\title{
Induced pluripotent stem cell-derived smooth muscle cells increase angiogenesis and accelerate diabetic wound healing
}

\author{
Jolanta Gorecka1', Xixiang Gao 1,2, Arash Fereydooni ${ }^{1}$, Biraja C Dash ${ }^{3}$, Jiesi Luo ${ }^{4,5,6,7}$, Shin \\ Rong Lee ${ }^{1}$, Ryosuke Taniguchi ${ }^{1}$, Henry C Hsia ${ }^{3}$, Yibing Qyang ${ }^{4,5,6,7}$ \& Alan Dardik ${ }^{* 1,8}$ \\ ${ }^{1}$ Vascular Biology \& Therapeutics Program \& The Department of Surgery, Yale School of Medicine, Yale University, New Haven, \\ CT 06519 USA \\ ${ }^{2}$ Department of Vascular Surgery, Xuanwu Hospital, Capital Medical University, Beijing, China \\ ${ }^{3}$ Section of Plastic Surgery, Department of Surgery, Yale School of Medicine, Yale University, New Haven, CT 06520, USA \\ ${ }^{4}$ Section of Cardiovascular Medicine, Department of Internal Medicine, Yale Cardiovascular Research Center, Yale School of \\ Medicine, New Haven, CT 06511, USA \\ ${ }^{5}$ Yale Stem Cell Center, Yale University, New Haven, CT 06520, USA \\ ${ }^{6}$ Vascular Biology \& Therapeutics Program, Yale School of Medicine, New Haven, CT 06519 USA \\ ${ }^{7}$ Department of Pathology, Yale University, New Haven, CT 06520, USA \\ ${ }^{8}$ Section of Vascular \& Endovascular Surgery, VA Connecticut Healthcare System, West Haven, CT 06516, USA \\ *Author for correspondence: alan.dardik@yale.edu
}

Aim: To assess the potential of human induced pluripotent stem cell-derived smooth muscle cells (hiPSCSMC) to accelerate diabetic wound healing. Methods: hiPSC-SMC were embedded in 3D collagen scaffolds and cultured in vitro for $72 \mathrm{~h}$; scaffolds were then applied to diabetic, nude mouse, splinted back wounds to assess in vivo healing. Cultured medium after scaffold incubation was collected and analyzed for expression of pro-angiogenic cytokines. Results: hiPSC-SMC secrete increased concentration of pro-angiogenic cytokines, compared with murine adipose derived stem cells. Delivery of hiPSC-SMC-containing collagen scaffolds accelerates diabetic wound healing and is associated with an increased number of total and M2 type macrophages. Conclusion: hiPSC-SMC promote angiogenesis and accelerate diabetic wound healing, making them a promising new candidate for treatment of diabetic wounds.

First draft submitted: 11 July 2019; Accepted for publication: 2 March 2020; Published online:

31 March 2020

Keywords: chronic wounds $\bullet$ diabetic foot ulcer $\bullet$ diabetic wounds $\bullet$ induced pluripotent stem cells $\bullet$ smooth muscle cells $\bullet$ stem cells 


\section{Graphical abstract:}

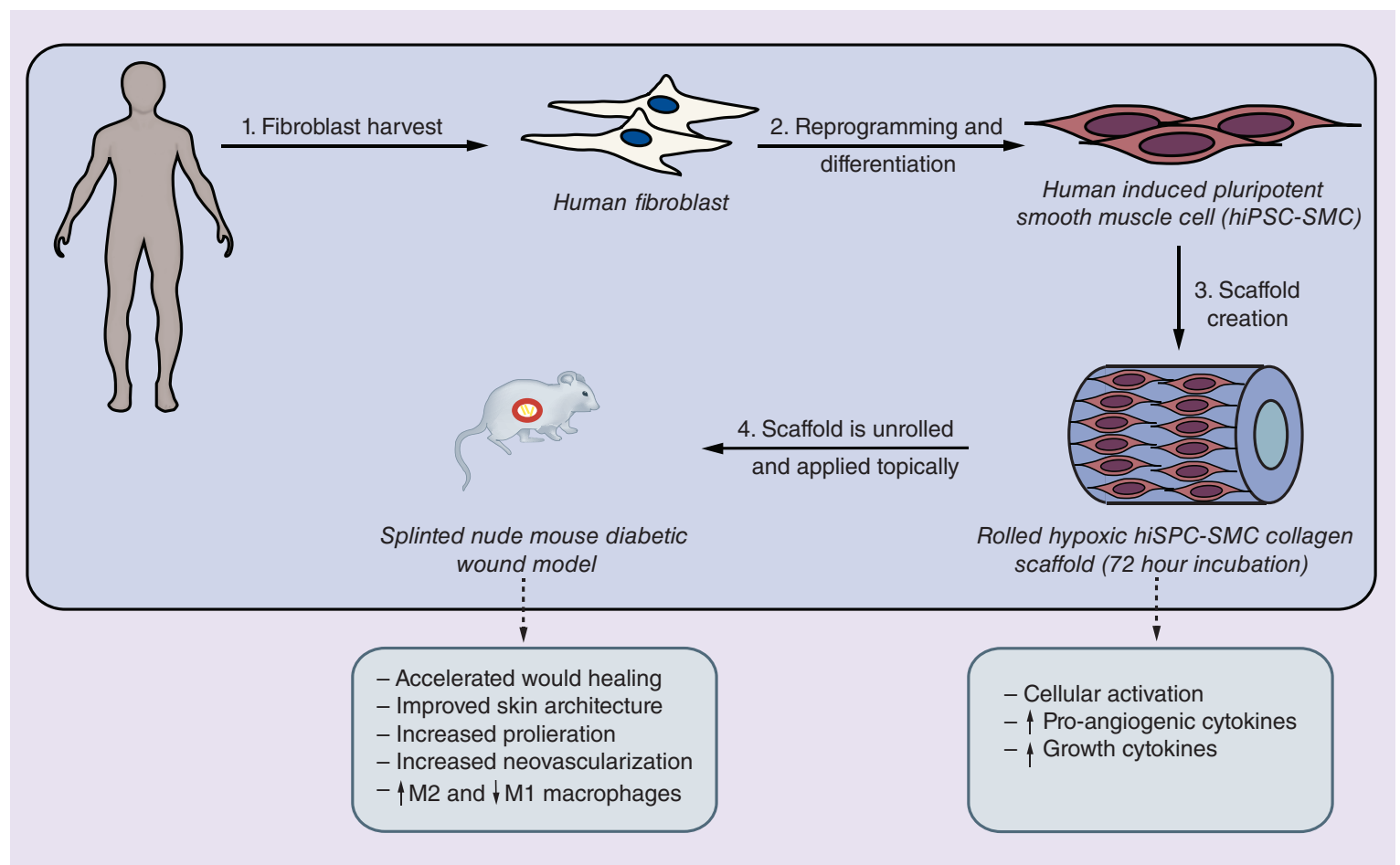

The epidemic of diabetes continues to worsen, with an estimated $9.4 \%$ of the US population affected in 2015, and of these individuals, over 6 million develop diabetic foot ulcers (DFU) annually $[1,2]$. This devastating complication is associated with 2.5-times increased risk of death within 5 years [3]. Standard treatment approaches for DFU include optimization of bloodflow, debridement, infection control and offloading [4]. Unfortunately, despite the best medical therapy, only $50 \%$ of DFU heal within 20 weeks and $50 \%$ recur within 18 months, highlighting the urgent need for improved therapies [5].

The pathophysiology of DFU includes excessive inflammation, decreased growth factor production, impaired angiogenesis, decreased macrophage and fibroblast function as well as impaired stem cell homing [6-9]. Stem cell therapy is emerging as a promising new treatment for DFU since stem cells may address some of the pathophysiological mechanisms underlying poor diabetic wound healing, including promoting cellular recruitment, decreasing inflammation, increasing extracellular matrix production, direct cellular replacement and increasing angiogenesis [10-12]. Adult-derived stem cells, such as mesenchymal stem cells (MSC) have shown efficacy in clinical trials and are currently available in several commercially available products [13-16]. However, in comparison to induced pluripotent stem cells (iPSC), adult-derived cells typically require more invasive harvesting techniques such as bone marrow or adipose tissue biopsies, are incapable of repopulating all dermal lineages and are delivered in an inactive state. Although MSC elaborate protrophic cytokines, MSC are strongly immunomodulatory and downregulate expression of inflammatory cytokines that may be necessary in the early stages of wound healing, [17] and thus MSC may not be the optimal cell type for clinical translation to human therapy for DFU.

Induced pluripotent stem cells are an exciting, clinically relevant candidate cell type for cell-based wound healing therapy. iPSC are pluripotent stem cells derived from adult somatic cells such as fibroblasts, obtained via noninvasive techniques such as skin biopsy and reprogrammed into a pluirpotent state in vitro [18,19]. iPSC can be differentiated into any adult cell lineage and their pool is essentially unlimited. Although iPSC hold great promise for multiple therapies, their clinical application is limited by their teratogenic potential when undifferentiated, potential genomic disruption during reprogramming and low differentiation efficiencies [18]. Thus far, human iPSC (hiPSC)-derived endothelial cells (hiPSC-EC), fibroblasts and hiPSC-derived MSC have shown accelerated wound healing in preclinical models [20-26]. In addition, codelivery of hiPSC-derived smooth muscle cells (hiPSCSMC) with hiPSC-EC increased neovascularization and improved wound healing compared with hiPSC-EC alone [27]. Similarly, hiPSC-derived early vascular cells, composed of a bicellular population of endothelial cells 
and pericytes, increased angiogenesis and led to accelerated healing, suggesting that hiPSC-derived vascular cells such as smooth muscle cells (SMC) may be an important cell type for translation to human therapy for DFU [28]. However, although methods to differentiate hiPSC into SMC are more efficient and yield a more uniform cellular population compared with methods to produce hiPSC-EC, no study thus far has examined the wound healing potential of hiPSC-SMC alone [29].

We previously showed that delivery of hypoxia-activated murine adipose-derived stem cells (ADSC) in a 3D collagen scaffold accelerates diabetic wound healing in a splinted full-thickness diabetic mouse wound model [30,31]. To increase the translational potential of these findings, we used this model to determine whether hiPSC-SMC alone accelerate wound healing. Since hiPSC-SMC enhance the pro-angiogenic and wound healing potential of hiPSC-EC, we hypothesized that hiPSC-SMC delivered alone in a 3D collagen scaffold promote angiogenesis and accelerate diabetic wound healing [27].

\section{Methods}

\section{Animals}

All animal studies were performed in compliance with federal guidelines and approved by Yale University's Institutional Animal Care and Use Committee. Male athymic nude mice (8-12 weeks; 20-30 g; Jackson Laboratory, ME, USA) were used for creation of the back wound model, as the prevalence of DFU is higher in males, globally [32]. To induce diabetes, mice were injected daily for 7 days with streptozotocin $(50 \mathrm{mg} / \mathrm{kg}$ IP; Tocris Biosciences, Bristol, UK). Blood glucose levels were measured with a handheld glucometer, starting 7 days after the final injection, via tail vein needle prick. Animals were considered to be diabetic after three consecutive blood glucose level measurements of $300 \mathrm{mg} / \mathrm{dl}$ or greater.

To create full thickness splinted back wounds, mice were anesthetized with vaporized isoflurane and two silicone splints with inner and outer diameters of 6 and $10 \mathrm{~mm}$, respectively, were sutured bilaterally on the upper dorsum of each animal. Six interrupted 5-0 polypropylene sutures were used to hold each splint in place. A full thickness (including panniculous carnosus) skin wound was then excised along the inner margin of the splint using scissors [33]. Collagen scaffolds (see below) were subsequently unrolled and divided into equal halves. Scaffolds were anchored to the wound bed with several interrupted 8-0 monofilament sutures immediately after wound creation. Scaffolds were left in the wound bed without removal for the duration of the experiment until day 10 or time of sacrifice. Wounds were covered with a semi-occlusive dressing (Tegaderm, 3M, MN, USA).

In some wounds, iPSC-SMC were directly injected without any scaffold placement; wounds were created as described above and 1 million cells suspended in $0.5 \mathrm{ml}$ cell culture medium were injected intradermally circumferentially around the wound.

Dressings were removed and wounds were examined every other day starting on postoperative day 0 . Mice were briefly anesthetized using isoflurane and wounds were photographed with an Olympus SP-800 UZ camera mounted on a tripod at a fixed distance of $7 \mathrm{~cm}$. Any loose sutures were removed and replaced to ensure splint was secured properly. Wound areas were then analyzed using ImageJ software (NIH, MD, USA). At the completion of the study, mice were euthanized and wounds were harvested and submerged in 10\% phosphate-buffered formalin overnight. The wound center was marked with black ink for orientation. The samples were then paraffin embedded, sectioned serially every $5 \mu \mathrm{m}$ and processed further as described below.

\section{hiPSC-SMC generation \& expansion}

The derivation of SMC from hiPSC was based upon an embryoid body (EB) formation-driven method, as previously described [34-36]. iPSC derived from human neonatal fibroblast cells were utilized for SMC differentiation. Briefly, hiPSC were expanded in mTeSR medium (Stem Cell Technologies, BC, Canada) under feeder-free culture conditions until $80 \%$ confluency and dissociated to form cell clusters with uniform sizes. Cell clusters were cultured in suspension within a 6-well low attachment plate in mTeSR medium for $24 \mathrm{~h}$. The culture medium was gradually transited to EB differentiation medium based on DMEM containing $10 \%$ of fetal bovine serum (FBS) to promote EB formation until day 6 . The EB were then transferred and seeded on a gelatin-coated plate for 6 days using EB differentiation medium. Subsequently, the differentiated cells were harvested, seeded on Matrigel-coated culture plate and cultured in smooth muscle growth medium (SmGM-2 medium, Lonza, MD, USA) for another 7-10 days. The hiPSC-derived SMC were further expanded in DMEM containing 10\% FBS for the generation of collagen scaffolds. 


\section{ADSC isolation \& expansion}

Murine ADSC were obtained from the abdominal fat of C57BL/6 mice, as previously described [30]. ADSC were cultured in EGM-2 media (Lonza, Basel, Switzerland), consisting of 10\% FBS (Gibco, MD, USA) and 1\% penicillin/streptomycin (Gibco), 2 mM L-glutamine (Corning Life Sciences, Corning, NY, USA) on plastic dishes at $37^{\circ} \mathrm{C}$ in $5 \% \mathrm{CO}_{2}$. Cell passages 2-6 were used in this study. ADSC identity was confirmed by FACS analysis of passage 2 cells [30]. ADSC identity was verified using anti-CD34, anti-CD90, anti-Sca-1, anti-CD105, anti-CD34 and anti-CD44 (BioLegend, CA, USA), using an LSRII flow cytometer (BD Biosciences, CA, USA).

\section{Collagen scaffolds}

Collagen scaffolds were created by mixing $500 \mu \mathrm{l} 10 \times$ minimum essential medium (Sigma-Aldrich, MO, USA) with $4 \mathrm{ml}$ type 1 rat tail collagen (Enzo Life Sciences, NY, USA) and adjusting the $\mathrm{pH}$ to 7.0 using drop wise addition of $1 \mathrm{M} \mathrm{NaOH}$ [30]. For cellular constructs, $2 \times 10^{6}$ cells in $0.5 \mathrm{ml}$ cell-specific culture medium were added to the collagen mixture prior to solidifying and $0.5 \mathrm{ml}$ cell-free phosphate-buffered saline (PBS) was used for acellular controls. The collagen solution was then allowed to solidify at room temperature within a rectangular mold ( $20 \mathrm{~min})$. The solidified gel was subsequently removed from the mold and compressed between glass plates $(5 \mathrm{~min})$ to remove all liquid. The remaining collagen sheet was then rolled using forceps and incubated in cell-specific, FBS-free, tissue culture media for $72 \mathrm{~h}$, in order to ensure maximal cellular activation in the scaffold periphery $\left(37^{\circ} \mathrm{C}\right)$. After a $72-\mathrm{h}$ incubation, tissue culture medium was collected for further processing and the rolled collagen scaffold was unrolled for in vivo studies.

\section{Angiogenesis array}

After a 72-h incubation of the rolled scaffold in exogenous growth factor-free medium, the medium was collected and used in the Proteome Profiler Angiogenesis Array (R\&D Systems, MN, USA), according to manufacturer's instructions. Briefly, nitrocellulose membranes were blocked in blocking buffer ( $1 \mathrm{~h}$ at room temperature). Samples were diluted in array buffer and mixed with detection antibody cocktail (biotin labeled against different proteins), followed by an overnight incubation on the membrane $\left(4^{\circ} \mathrm{C}\right)$. Following overnight incubation, membranes were washed three-times in wash buffer and subsequently incubated in horseradish peroxidase (HRP)-conjugated streptavidin complex for $30 \mathrm{~min}$ (room temperature). After washing, signal was detected by electrochemiluminescence system (GE Healthcare, IL, USA). The signal density of each blot was then determined by densitometry using ImageJ software (NIH, MD, USA).

\section{ELISA}

After a 72-h incubation of the rolled scaffold in exogenous growth factor-free medium, the medium was collected and used in the Quantikine human and mouse VEGF-A, bFGF, TGF- $\beta 1$ and IGF-1 ELISA kits (R\&D Systems); samples were diluted per manufacturer's suggestion. Standards, controls and samples were loaded into a 96-well plate coated with protein specific antibodies. Samples were incubated for $2 \mathrm{~h}$ (room temperature), followed by three washes. Protein-specific conjugated antibodies were then added to the wells and incubated for another $2 \mathrm{~h}$ (room temperature). After washing, wells were incubated in substrate solution (20 $\mathrm{min}$ at room temperature) and protein concentrations were measured with a microplate reader set to $450 \mathrm{~nm}$.

\section{Immunohistochemistry \& immunofluorescence}

Immunohistochemistry and immunofluorescence were performed as previously described [30]. Briefly, slides were de-paraffined using xylene and a graded series of ethanol washes. Sections were heated in citric acid buffer ( $\mathrm{pH}$ 6.0) at $100^{\circ} \mathrm{C}$ for $10 \mathrm{~min}$ for antigen retrieval and subsequently blocked with $5 \%$ bovine serum albumin in PBS containing Tween (PBST) for $1 \mathrm{~h}$ at room temperature prior to an overnight incubation at $4^{\circ} \mathrm{C}$ with the primary antibodies diluted in PBST.

Primary antibodies used for immunohistochemistry include: anti-Ki67 (rabbit; 1:500; Abcam), anti-caspase 3 (rabbit; 1:500; Cell Signaling, MA, USA), anti-VEGF-A (rabbit; 1:250; Santa Cruz Biotechnology), anti-bFGF (mouse; 1:100; BioLegend), anti-HLA class 1 (mouse; 1:100; Abcam), anti-CD31 (rabbit; 1:200; Cell Signaling), anti-hypoxia inducible factor- $1 \alpha$ (HIF-1 $\alpha$; rabbit; 1:100; Novus Biologicals, CO, USA). For immunohistochemistry, after incubation with primary antibodies overnight, sections were treated with Envision+ dual link HRP rabbit/mouse secondary antibodies at room temperature for $1 \mathrm{~h}$ (Agilent Dako, CA, USA). After developing with 
diaminobenzidine tetrahydrochloride and counterstaining with hematoxylin, photomicrographs were obtained and positive cells were manually counted and reported as a percentage of total cells in four high-power fields.

Primary antibodies used for immunofluorescence include: anti-elastin (mouse; 1:100; Santa Cruz Biotechnology), anti-smooth muscle-myosin heavy chain (SM-MHC; mouse; 1:100; Abcam), anti- $\alpha$-smooth muscle actin (SMA; rabbit: 1:100; Abcam), anti-HLA (rat; 1:200 Santa Cruz Biotechnology), anti-L-caldesmon (mouse; 1:200; Santa Cruz Biotechnology), anti-CD68 (mouse; 1:200; BioRad, PA, USA), anti-inducible nitric oxide synthase (iNOS rabbit; 1:200; Cell Signaling), anti-TNF $\alpha$ (rabbit; 1:200; Abcam), anti-CD206 (mouse; 1:200; BioRad) and anti-TGM2 (rabbit; 1:200; Cell Signaling). For immunofluorescence, after incubation with primary antibodies overnight, sections were incubated with secondary antibodies at room temperature for $1 \mathrm{~h}$ using goat anti-rabbit Alexa Fluor 568 (Life Technologies, NY, USA), goat anti-mouse Alexa-Fluor-568 (Life Technologies), goat anti-chicken Alexa-Fluor-568 (Life Technologies), goat anti-rat Alexa-Fluor-568 (Life Technologies) or goat anti-rat Alexa-Fluor-488 (Life Technologies). Sections were then stained with SlowFade ${ }^{\circledR}$ Gold Antifade Mountant with DAPI (Life Technologies) and a coverslip was applied. Digital fluorescence images were captured and positive cells were manually counted in ImageJ software (NIH).

For cell staining, cells were cultured under standard conditions in a 12-well plate on glass coverslips until approximately 50\% confluence, at which time they were fixed with $4 \%$ paraformaldehyde (30 min, room temperature) and processed as described above.

For scaffold staining, scaffolds were unrolled, fixed in $4 \%$ paraformaldehyde (10 $\mathrm{min}$, room temperature) and stained in a 24-well plate. Scaffolds were washed in PBST and blocked in 5\% bovine serum albumin in PBST. They were subsequently incubated in primary antibodies overnight at $4{ }^{\circ} \mathrm{C}$ with the primary antibodies diluted in PBST. Following incubation with primary antibodies overnight, scaffolds were treated with Envision+ dual link HRP rabbit/mouse secondary antibodies at room temperature for $1 \mathrm{~h}$ (Agilent Dako). After developing with diaminobenzidine tetrahydrochloride and counterstaining with hematoxylin, scaffolds were mounted onto slides and dehydrated through a graded series of ethanols. Photomicrographs were subsequently obtained and positive cells were manually counted and reported as a percentage of total cells in four high-power fields.

\section{Flow cytometry}

Harvested cells were fixed using 2\% paraformaldehyde (30 min, room temperature) and washed with PBS threetimes, followed by blocking with $5 \%$ bovine serum albumin and $0.1 \%$ Triton-X-100. The primary antibodies anti-SM-22 $\alpha$ (Abcam) and anti-calponin (Sigma, MO, USA) were used in a concentration of $1 \mu \mathrm{g} / \mathrm{ml}$. Mouse and goat IgG isotype controls (Thermo Fisher, MA, USA; Santa Cruz Biotechnology) were used as control. Cell fluorescence was measured by a flow cytometer (BD, LSR II) and cell cycle analysis was performed using FlowJo software.

\section{Statistical analysis}

Data are expressed as mean \pm standard error of the mean (SEM). Statistical analysis was performed using Student's t-test to compare two groups or analysis of variance followed by Tukey's post hoc test for multiple comparisons, using Graphpad Prism version 7.0 (La Jolla, CA, USA). A p-value $<0.05$ was considered significant.

\section{Results}

\section{hiPSC-SMC have a contractile phenotype in vitro}

We examined the activity of hiPSC-SMC in a diabetic nude mouse splinted back wound model. Human iPSC were differentiated into SMC in vitro [34]; hiPSC-SMC displayed a predominantly contractile phenotype, with strong intensity of elastin (100\%), SM-MHC (100\%) and SMA (97\%), markers of the contractile phenotype (Figure 1A $\&$ B). hiPSC-SMC displayed low intensity of the synthetic smooth muscle phenotype markers caldesmon light chain $(22 \%)$ and vimentin $(7 \%)$, also consistent with a predominantly contractile phenotype (Figure 1C \& D). We confirmed these findings with FACS analysis of two additional contractile markers that showed $93 \%$ of hiPSC-SMC were positive for calponin and 91\% of hiPSC-SMC were positive for SM-22 $\alpha$ (Figure 1E \& F). These data show that prior to incorporation into collagen scaffolds, iPSC-SMC were differentiated and displayed a contractile SMC phenotype in vitro. 

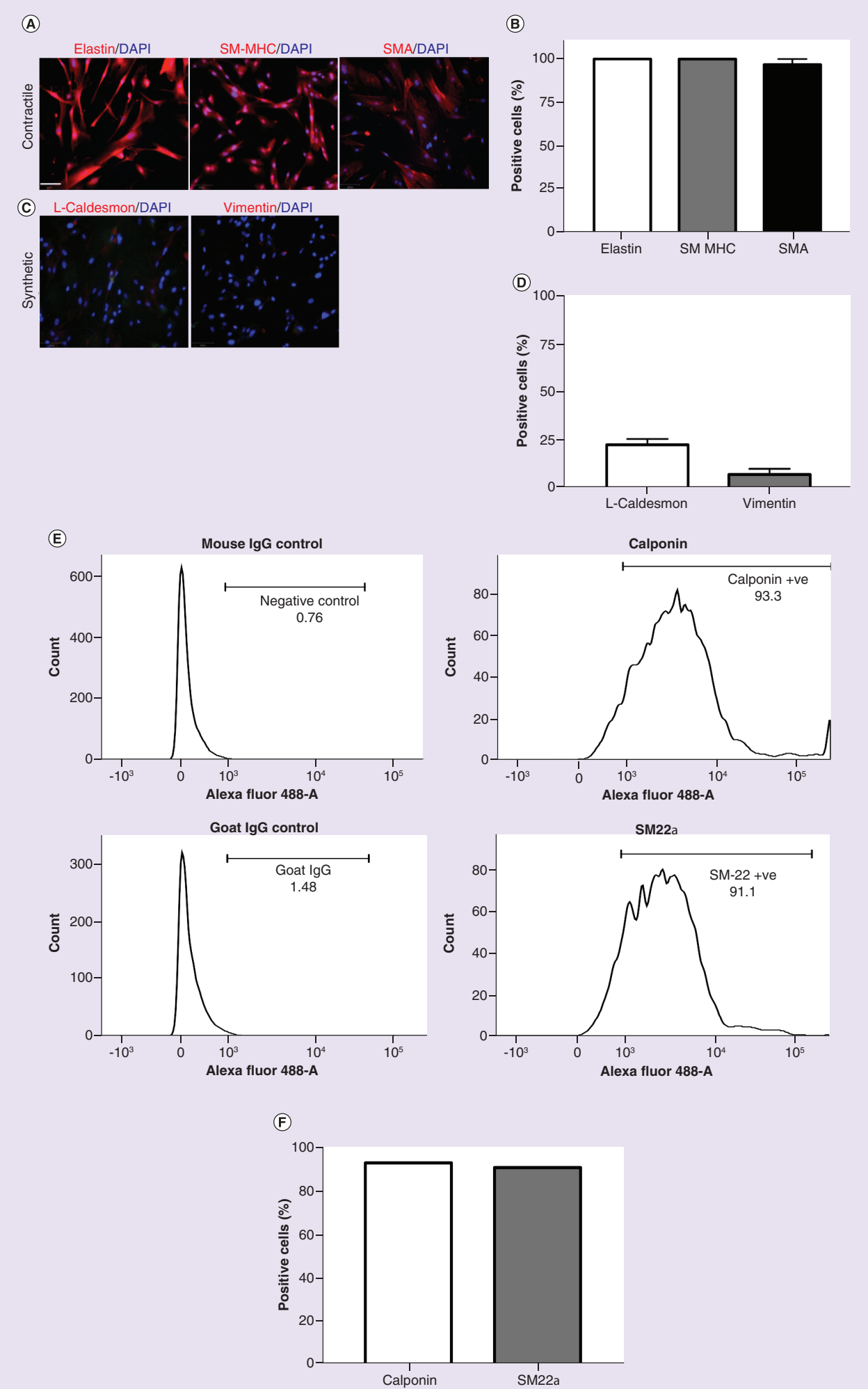

Figure 1. Characterization of human-induced pluripotent stem cell-derived smooth muscle cells. (A) Immunofluorescence images of cells in tissue culture stained with contractile smooth muscle cell markers elastin (left), SM-MHC; (middle) and SMA (right); DAPI, blue; scale bar represents $100 \mu \mathrm{m}$. (B) Bar graph representing the percentage of hiPSC-SMC-positive for elastin, SM-MHC and SMA; $\mathrm{n}=4$. (C) Immunofluorescence images of cells in tissue culture stained with synthetic smooth muscle cell markers including L-caldesmon (left) and vimentin (right); DAPI, blue. (D) Bar graph representing the percentage of hiPSC-SMC-positive for L-caldesmon and vimentin; $\mathrm{n}=4$. (E) FACS analysis of hiPSC-SMC for calponin (upper row) and smooth muscle-22 alpha (SM-22 $\alpha$, lower row). (F) Bar graph representing percentage of hiPSC-SMC positive for calponin or SM-22 $\alpha$.

DAPI: 4',6-diamidino-2-phenylindole; FACS: Fluorescence-activated cell sorting; hiPSC-SMC: Human-induced pluripotent stem cell-derived smooth muscle cell; SM-MHC: Smooth muscle-myosin heavy chain; SMA: Smooth muscle actin. 


\section{hiPSC-SMC release increased angiogenic \& regenerative cytokines compared with ADSC}

We determined the angiogenic profile of hiPSC-SMC in collagen scaffolds and used ADSC as positive controls. Cells were incorporated into a rolled collagen scaffold that was incubated for $72 \mathrm{~h}$ in exogenous growth factor-free medium and then subsequently analyzed; scaffolds were rolled prior to incubation to mimic the natural hypoxic stem cell niche, leading to cellular activation [30]. Using a commercially available angiogenesis antibody array, hiPSC-SMC released into the medium a significantly larger concentration of 28 out of 31 angiogenic cytokines compared with ADSC (Figure 2A \& B); medium from acellular collagen scaffolds contained undetectable levels of all 31 examined cytokines (Figure 2A).

To confirm these results and to determine whether rolled scaffolds activate hiPSC-SMC compared with flat scaffolds, cell culture medium was collected from rolled acellular and ADSC, flat and rolled hiPSC-SMC and rolled primary human coronary artery SMC-containing scaffolds and assessed for several growth factors that regulate angiogenesis. hiPSC-SMC secreted a significantly higher concentration of VEGF-A compared with ADSC and flat scaffolds containing hiPSC-SMC, with no difference in VEGF-A concentration between induced and primary SMC ( $<<0.0001$; Figure 2C). The concentration of bFGF, a fibroblast chemoattractant, was highest in the hiPSC-SMC group $(\mathrm{p}<0.0001)$, to an even greater amount than primary SMC $(\mathrm{p}<0.0001$; Figure $2 \mathrm{D})$. The concentration of TGF- $\beta 1$ was higher in all SMC groups compared with ADSC-containing scaffolds $(p<0.0001)$ and there was no difference between flat and rolled scaffolds ( $\mathrm{p}=0.1452)$, nor between hiPSC-SMC and primary SMC ( $\mathrm{p}=0.087$; Figure 2E). However, there was no significant difference in concentration of IGF-1, a stimulator of mitogenesis and cell survival, between all cellular groups (Figure $2 \mathrm{~F}$ ). To determine whether rolled scaffolds induce hypoxia, we compared the expression of HIF- $1 \alpha$ in flat and rolled scaffolds at 0,48 and $72 \mathrm{~h}$ of incubation in vitro. We found that the expression of HIF-1 $\alpha$ in cultured cells increase in rolled, but not flat, scaffolds, over time ( $\mathrm{p}<0.0001$; Figure $2 \mathrm{G}$ ). These results suggest that hiPSC-SMC are activated by the hypoxic environment inside the rolled scaffold and secrete an increased amount of angiogenic and regenerative cytokines compared with murine ADSC.

\section{hiPSC-SMC accelerate diabetic wound healing}

To determine whether use of hiPSC-SMC in our previously described rolled collagen scaffold model accelerates wound healing, we applied acellular, ADSC or hiPSC-SMC-containing collagen scaffolds to full thickness cutaneous back wounds in diabetic nude mice; we used a splinted wound model to prevent healing secondary to contraction [33]. Murine ADSCs were used as a positive control as they have previously been investigated and show wound healing in our diabetic splinted wound model [30]. Scaffolds were incubated in vitro for $72 \mathrm{~h}$ in rolled configuration and then unrolled for subsequent application in vivo [30]. hiPSC-SMCs accelerated wound healing compared with acellular and ADSC-containing scaffolds ( $\mathrm{p}<0.0001$; Figure $3 \mathrm{~A} \& \mathrm{~B}$ ), with differences detectable as early as day 2 . At the leading wound edge hiPSC-SMC-treated wounds were associated with significantly thicker epidermal $(\mathrm{p}<0.0001$; Figure 3C \& D) and muscle layers ( $\mathrm{p}=0.0009$; Figure $3 \mathrm{E} \& \mathrm{~F}$ ) compared with both acellular and ADSC-treated wounds. These results suggest that in addition to accelerated wound healing, hiPSC-SMC treatment is associated with epidermal and muscle thickening, suggesting improved wound architecture.

Wounds were excised and examined at 7 days; wounds treated with hiPSC-SMC were associated with a significantly higher rate of proliferation ( $<<0.0001$; Figure 4A \& B); as expected, apoptosis was uniformly low, with no significant differences between groups ( $<<0.3786$; Figure $4 \mathrm{C} \& \mathrm{D}$ ). Wounds were also examined at 4 days; wounds treated with hiPSC-SMC were associated with a higher percentage of both VEGF-A $(\mathrm{p}=0.0005$; Figure 4E \& F) and bFGF-positive cells $(\mathrm{p}=0.0172$; Figure 4G \& H). Wounds treated with hiPSC-SMC were also associated with increased number of CD31-positive cells $(\mathrm{p}=0.0002$; Figure $4 \mathrm{I} \& \mathrm{~J})$. These data show that wounds treated hiPSC-SMC had increased cellular proliferation, expression of pro-angiogenic and regenerative cytokines and angiogenesis in vivo.

We determined the numbers of macrophages as well as macrophage phenotype associated with each treatment group. hiPSC-SMC treatment was associated with increased number of macrophages compared with acellular and ADSC-treated groups $(\mathrm{p}=0.0004$; Figure 5A \& B). There were fewer numbers of CD68/TNF $\alpha$ dual-positive cells as well as CD68/iNOS dual-positive cells in wounds treated with hiPSC-SMC compared with acellular $(\mathrm{p}=0.0008)$ and ADSC treatment $(\mathrm{p}=0.0375$; Figure 5C-F), suggesting fewer M1 type macrophages. However, there were increased numbers of CD68/CD206 dual-positive cells as well as CD68/TGM2 dual-positive cells in wounds treated with hiPSC-SMC compared with ADSC $(\mathrm{p}=0.0214$; Figure 5G-J), consistent with increased numbers of M2 type macrophages. These results suggest that treatment of wounds with hiPSC-SMC is associated 


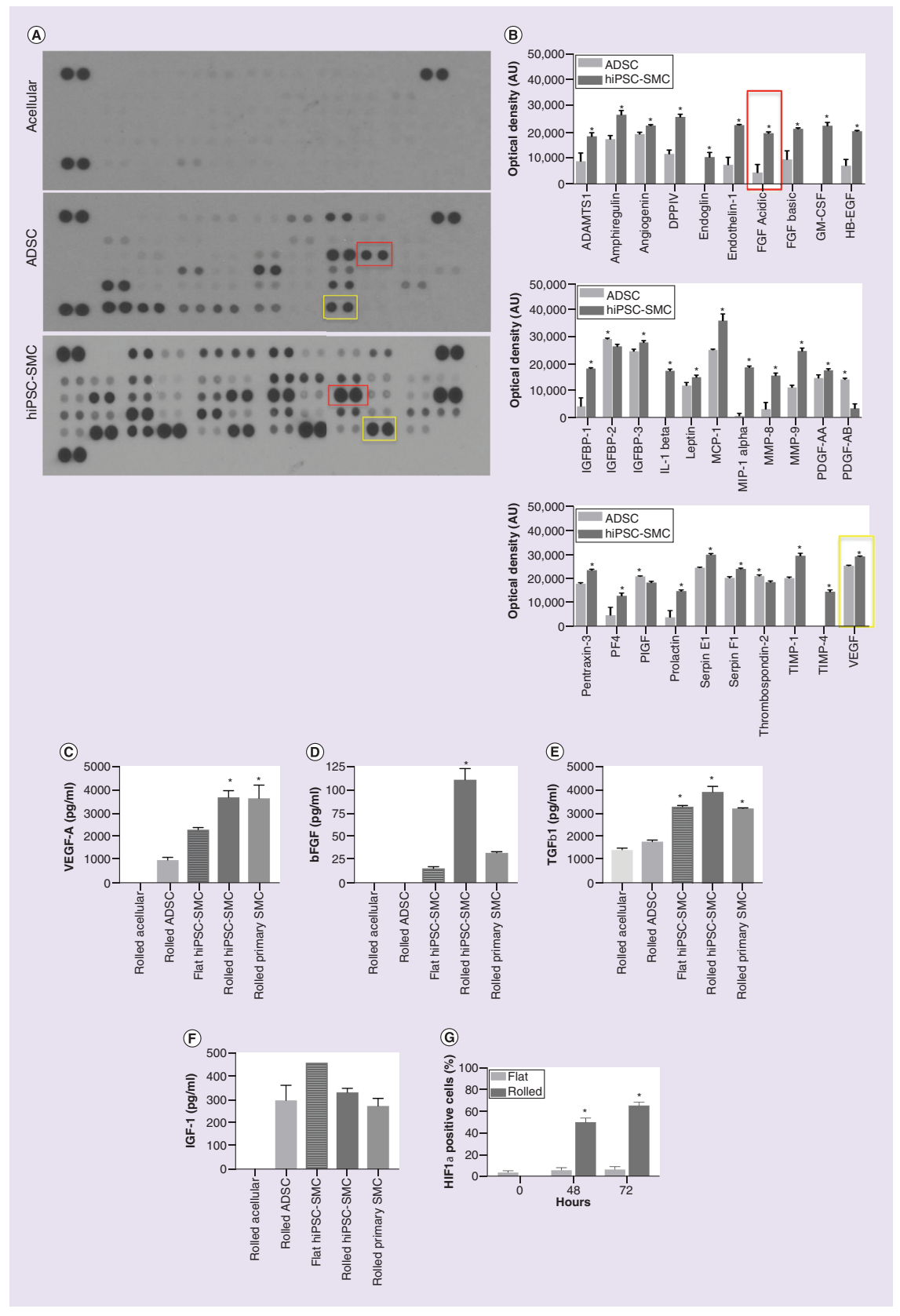

Figure 2. Human-induced pluripotent stem cell-derived smooth muscle cells secrete increased amounts of angiogenic and regenerative cytokines compared with adipose-derived stem cells. (A) Representative membrane images of angiogenesis antibody array using conditioned medium from acellular (top), ADSC (middle) or hiPSC-SMC (bottom) rolled scaffolds, after a 72-h incubation in vitro; yellow boxes encircle pair of VEGF blots and red boxes encircle pair of FGF-acidic blots. (B) Bar graphs representing cytokines with significantly different concentrations between ADSC and hiPSC-SMC groups; mean \pm standard error of the mean; * $p<0.05$ (t-test); $n=3$. Yellow box highlights VEGF and red box highlights acidic FGF and correspond to similarly colored boxes in panel A. No analysis was performed on the acellular scaffold group as all cytokines were below detectable levels. (C) Bar graph representing concentration of VEGF-A in culture medium after a 72-h incubation of acellular, ADSC, flat hiPSC-SMC, rolled hiPSC-SMC and primary SMC-containing scaffolds; ${ }^{*} \mathrm{p}<0.0001$ (ANOVA); ${ }^{*}<0.0001$ ADSCs vs hiPSC; ${ }^{*} p=0.0013$ flat vs rolled hiPSC-SMC; * $p>0.999$ hiPSC-SMC vs primary SMC (Tukey's post hoc); $\mathrm{n}=2-7$. (D) Bar graph representing concentration of bFGF in culture medium after a 72-h incubation of acellular, ADSC, flat hiPSC-SMC, rolled hiPSC-SMC and primary SMC containing scaffolds; * $p<0.0001$ (ANOVA); $p<0.0001$ ADSCs vs hiPSC; * $p<0.0001$ flat vs rolled hiPSC-SMC; ${ }^{*} \mathrm{p}<0.0001$ hiPSC-SMC vs primary SMC (Tukey's post hoc); $\mathrm{n}=2-6$. (E) Bar graph representing concentration of TGF- $\beta 1$ in culture medium after a 72-h incubation of acellular, ADSC, flat hiPSC-SMC, rolled hiPSC-SMC and primary SMC-containing Scaffolds; ${ }^{*} p<0.0001$ (ANOVA); $p<0.0001$ ADSC vs hiPSC; $p=0.1452$ flat vs rolled hiPSC-SMC; ${ }^{*} p=0.087$ hiPSC-SMC vs primary SMC (Tukey's post hoc); $\mathrm{n}=2-5$. (F) Bar graph representing concentration of IGF-1 in culture medium after a 72-h incubation of acellular, ADSC, flat hiPSC-SMC, rolled hiPSC-SMC and primary SMC-containing scaffolds; $p<0.0001$ (ANOVA); $p=0.9484$ ADSC vs hiPSC-SMC; $p=0.2950$ flat vs rolled hiPSC-SMC; $p=0.8790$ hiPSC-SMC vs primary SMC (Tukey's post hoc); $n=2-7$. (G) Bar graph representing the number of cells expressing HIF-1 $\alpha$ in flat vs rolled scaffolds at 0,48 and $72 \mathrm{~h}$ after incubation; $p<0.0001$ (two-way ANOVA); $p<0.0001$ flat vs rolled at $48 \mathrm{~h} ; \mathrm{p}<0.0001$ flat vs rolled at $72 \mathrm{~h}$ (Tukey's post hoc); $\mathrm{n}=3$.

ADSC: Adipose-derived stem cell; ANOVA: Analysis of variance; HIF1- $\alpha$ : Hypoxia inducible factor-1 $\alpha$; hiPSC-SMC: Human-induced pluripotent stem cell-derived smooth muscle cell; SMC: Smooth muscle cell. 
(A)

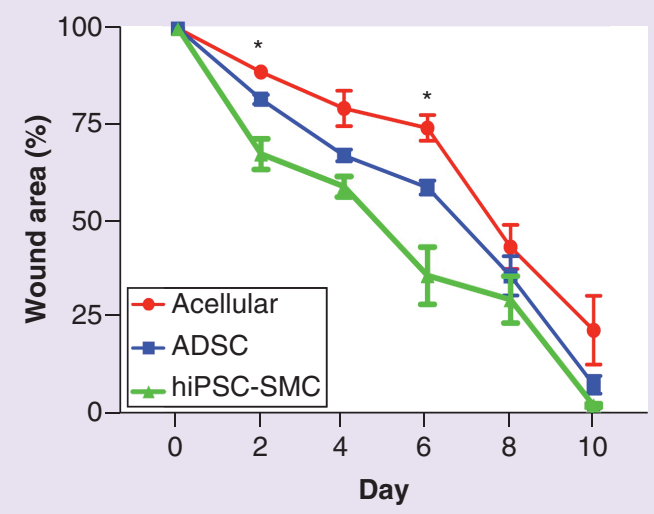

(C) Acellular

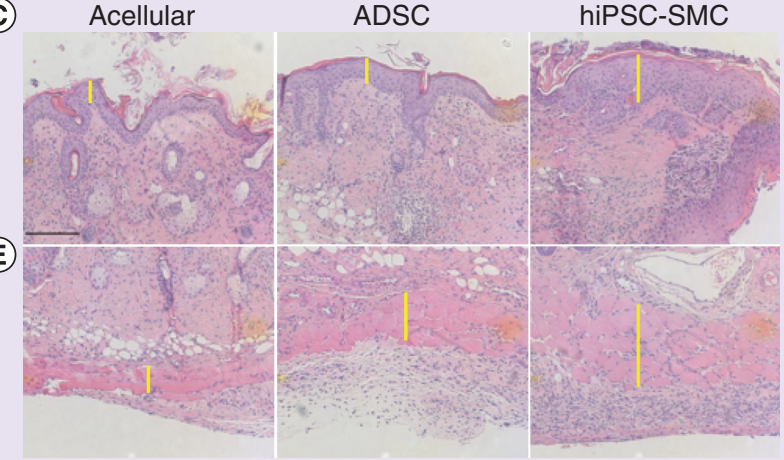

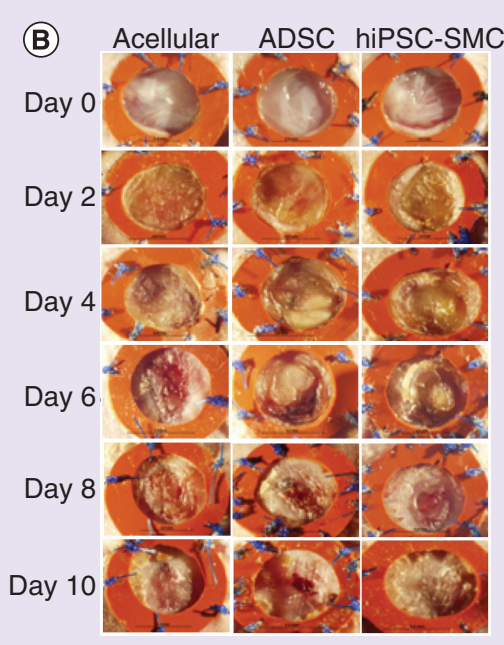

(D)

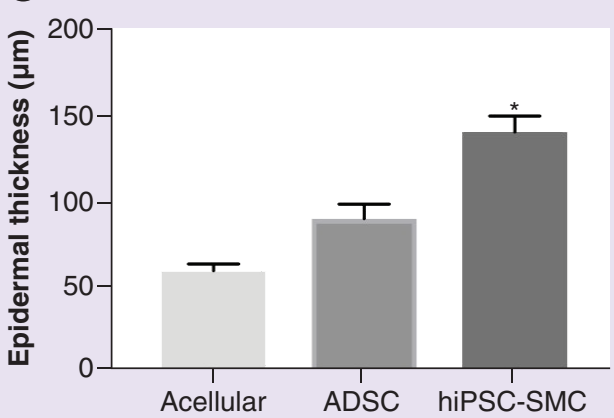

(F)

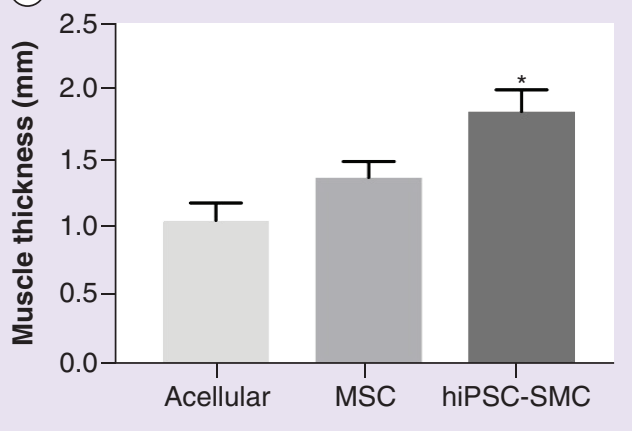

Figure 3. Human-induced pluripotent stem cell-derived smooth muscle cell accelerate diabetic wound healing to a greater extent than acellular and adipose-derived stem cell-containing scaffolds. (A) Line graph representing wound area over time in acellular, ADSC and hiPSC-SMC-treated groups; ${ }^{*} p<0.0001$ (two-way ANOVA); $p=0.0013$ at day 2 acellular vs hiPSC-SMC; ${ }^{*} p 0.0001$ at day 6 acellular vs hiPSC-SMC and ${ }^{*} p=0.0014$ at day 6 ADSC vs hiPSC-SMC (Tukey's post hoc); $n=4-6$. (B) Photographs of representative wounds at days 0, 2, 4, 6, 8 and 10 in acellular, ADSC and hiPSC-SMC groups; scale bar, $5 \mathrm{~mm}$. (C) Photomicrographs of hematoxylin and eosin-stained epidermal samples from leading wound edge excised at 4 days in acellular, ADSC and hiPSC-SMC groups; scale bar, $150 \mu \mathrm{m}$; yellow line shows area of maximal thickness. (D) Bar graph showing epidermal thickness in acellular, ADSC and hiPSC-SMC groups at 4 days; ${ }^{*} p<0.0001$ (ANOVA); ${ }^{*} p=0.0003$ ADSC vs hiPSC-SMC (Tukey's post hoc); $n=3-5$. (E) Photomicrographs of hematoxylin and eosin-stained muscle layers from leading wound edge excised at 4 days in acellular, ADSC and hiPSC-SMC groups; scale bar, $150 \mu \mathrm{m}$; yellow line shows area of maximal thickness. (F) Bar graph showing muscle thickness in acellular, ADSC and hiPSC-SMC groups at 4 days; ${ }^{*} p<0.0009$ (ANOVA); $p=0.0471$ ADSC vs hiPSC-SMC (Tukey's post hoc); $\mathrm{n}=3-5$.

ADSC: Adipose-derived stem cell; ANOVA: Analysis of variance; hiPSC-SMC: Human-induced pluripotent stem cell-derived smooth muscle cell. 


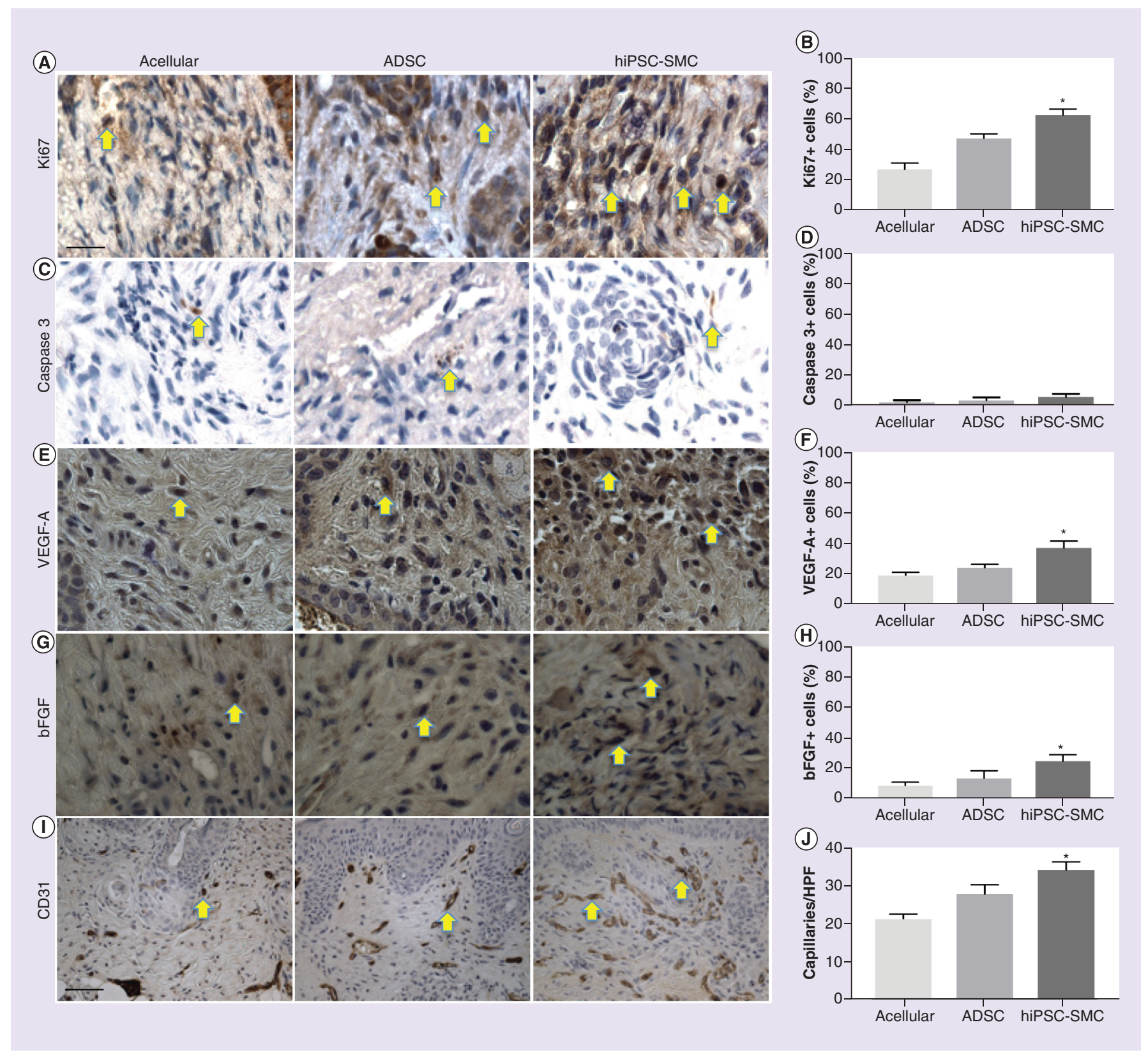

Figure 4. Human-induced pluripotent stem cell-derived smooth muscle cells are associated with increased proliferation, cytokine secretion and angiogenesis in vivo. (A) Representative photomicrographs of skin samples (7 days) stained for Ki67 in acellular, ADSC and hiPSC-SMC groups; scale bar, $100 \mu \mathrm{m}$; yellow arrows denote positive cells. (B) Bar graph showing percentage of cells positive for Ki67; ${ }^{*} \mathrm{p}<0.0001$ (ANOVA); $n=4$. (C) Representative photomicrographs of skin samples (7 days) stained for caspase-3 in acellular, ADSC and hiPSC-SMC groups; yellow arrows denote positive cells. (D) Bar graph showing percentage of cells positive for caspase-3; ${ }^{*} \mathrm{p}=0.3786$ (ANOVA); $n=4$. (E) Representative photomicrographs of skin samples (4 days) stained for VEGF-A in acellular, ADSC and hiPSC-SMC groups; yellow arrows denote positive cells. (F) Bar graph showing percentage of cells positive for VEGF-A in acellular, ADSC and hiPSC-MSC groups; ${ }^{*} p=0.0005$ (ANOVA); $n=3$. (G) Representative photomicrographs of skin samples (4 days) stained for bFGF in acellular, ADSC and hiPSC-SMC groups; yellow arrows denote positive cells. (H) Bar graph showing percentage of cells positive for bFGF; ${ }^{*} \mathrm{p}=0.0172$ (ANOVA); $n=4-6$. (I) Representative photomicrographs of skin samples (4 days) stained for CD31 in acellular, ADSC and hiPSC-SMC groups; scale bar, $250 \mu \mathrm{m}$; yellow arrows denote positive cells. (J) Bar graph showing capillary number per high power field in acellular, ADSC and hiPSC-SMC groups; * $\mathrm{p}=0.0002$ (ANOVA); $\mathrm{n}=4$

ADSC: Adipose-derived stem cell; ANOVA: Analysis of variance; hiPSC-MSC: Human-induced pluripotent stem cell-derived MSC; hiPSC-SMC: Human-induced pluripotent stem cell-derived smooth muscle cell. 

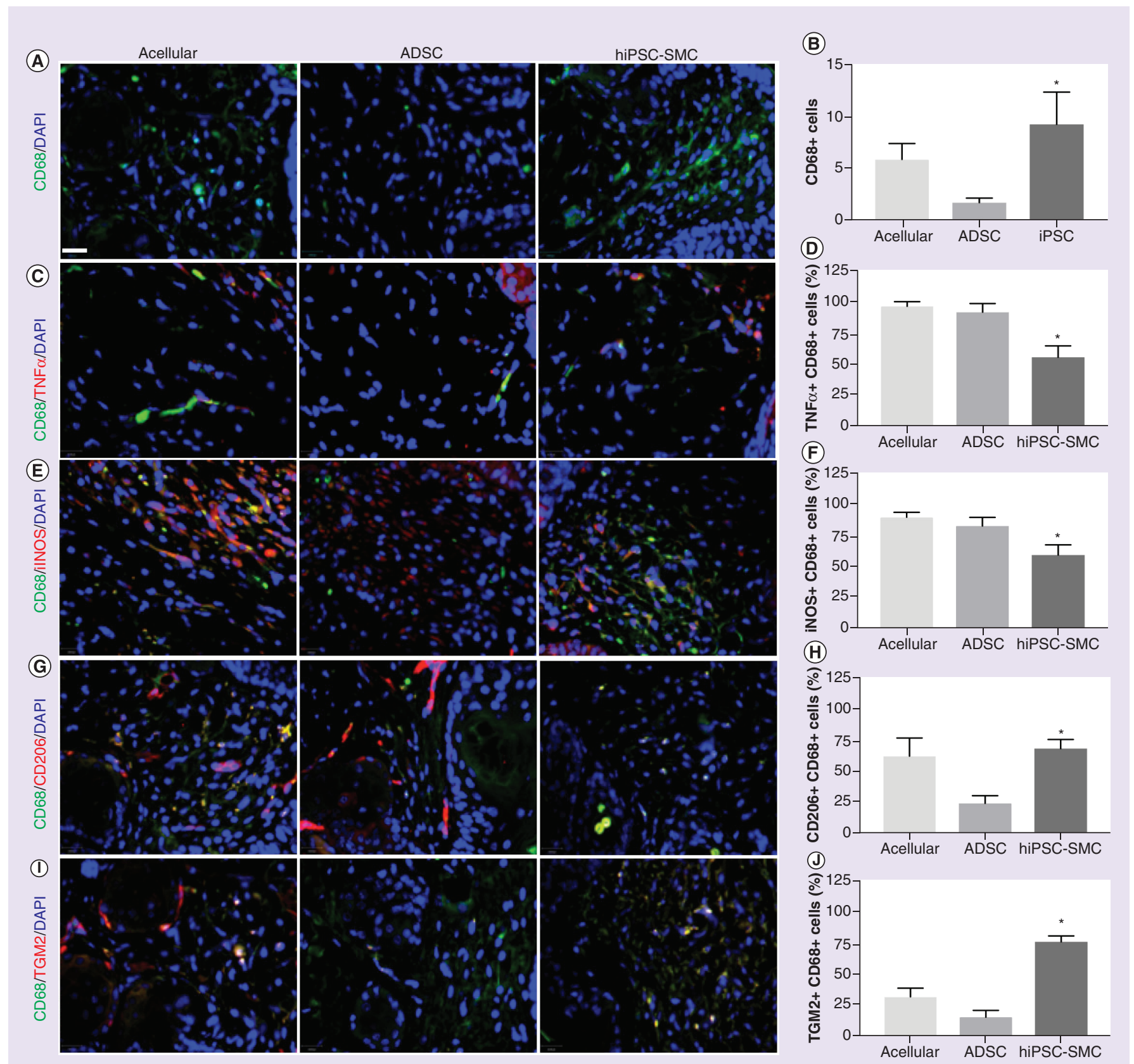

Figure 5. Human-induced pluripotent stem cell-derived smooth muscle cell-treated wounds show decreased M1-type macrophages and increased M2-type macrophages. (A) Immunofluorescence of excised wounds (4 days) showing CD68-positive cells (green) in acellular (left), ADSC (middle) and hiPSC-SMC (right) groups; DAPI, blue; scale bar, $20 \mu \mathrm{m}$. (B) Bar graph showing the number of CD68-positive cells per high power field in acellular, ADSC and hiPSC-SMC groups; ${ }^{*}=0.0004$ (ANOVA); $n=3$. (C) Immunofluorescence of excised wounds (4 days) showing immunoreactivity with CD68 (green) and TNF $\alpha$ (red); DAPI, blue. (D) Bar graph showing percentage of TNF $\alpha / C D 68$ dual-positive cells; ${ }^{*} p=0.0008$ (ANOVA); $n=4-6$. (E) Immunofluorescence of excised wounds (4 days) showing immunoreactivity with CD68 (green) and iNOS (red); DAPI, blue. (F) Bar graph showing percentage of iNOS/CD68 dual-positive cells; * $p=0.0087$ (ANOVA); $\mathrm{n}=$ 4-6. (G) Immunofluorescence of excised wounds (4 days) showing immunoreactivity with CD68 (green) and CD206 (red); DAPI, blue. (H) Bar graph showing percentage of CD68/CD206 dual-positive cells; ${ }^{*} p=0.0214$ (ANOVA); $n=4-6$. (I) Immunofluorescence of excised wounds (4 days) showing immunoreactivity with CD68 (green) and TGM2 (red); DAPI, blue. (J) Bar graph showing percentage of CD68/TGM2 dual-positive cells; * $p<0.0001$ (ANOVA); $n=4-6$.

ADSC: Adipose-derived stem cell; ANOVA: Analysis of variance; DAPI: 4',6-diamidino-2-phenylindole; hiPSC-SMC: Human-induced pluripotent stem cell-derived smooth muscle cell. 


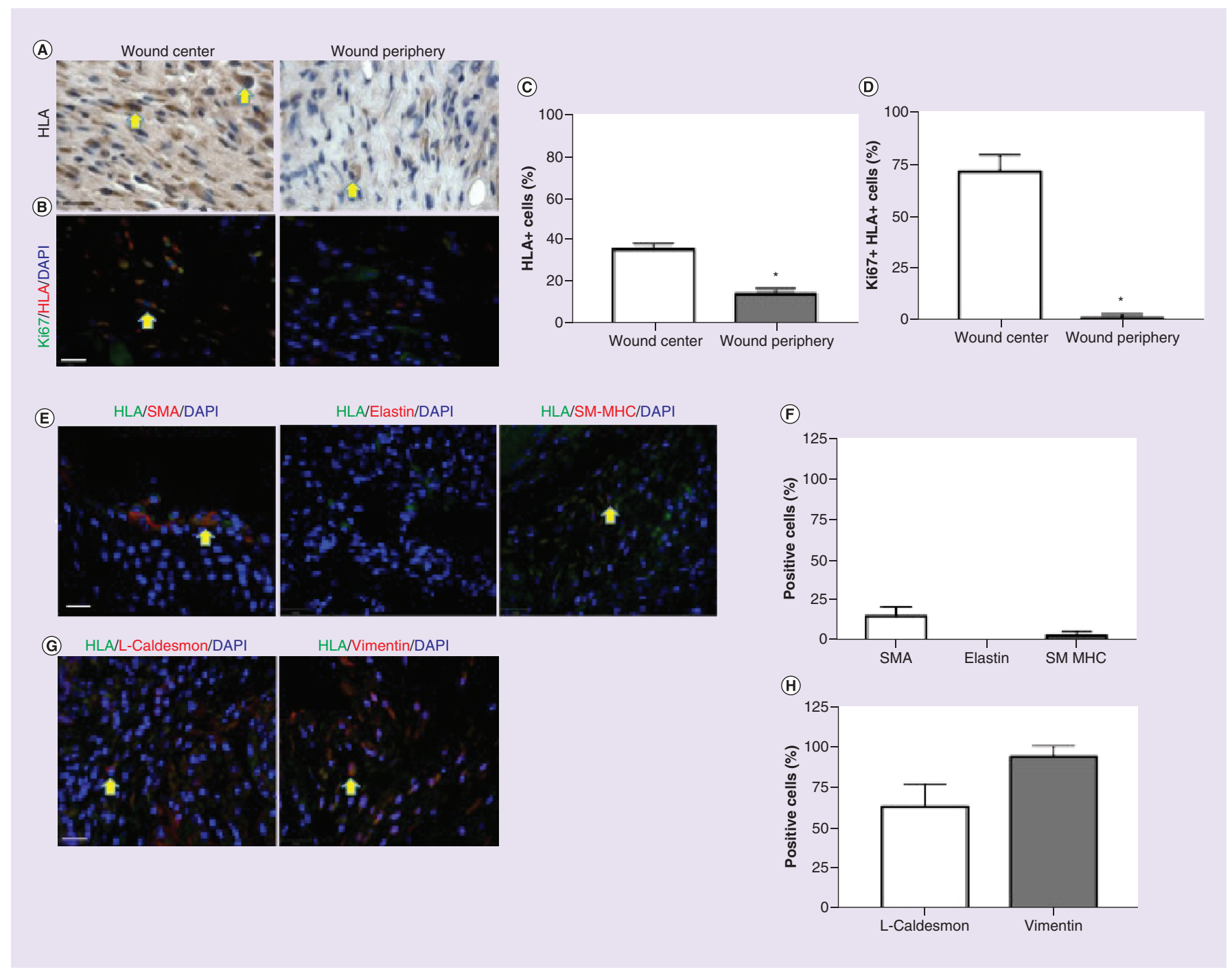

Figure 6. Human-induced pluripotent stem cell-derived smooth muscle cell are present in wounds at 7 days. (A) Photomicrographs of excised wounds stained for HLA, in the wound center (left) or periphery (right); scale bar, $10 \mu$ m; yellow arrows show positive cells. (B) Immunofluorescence of excised wounds showing immunoreactivity for both HLA (red) and Ki67 (green) in the wound center (left) or periphery (right); DAPI, blue; scale bar, $30 \mu \mathrm{m}$; yellow arrows show positive cells. (C) Bar graph showing the percent of HLA-positive cells in the wound center or periphery; ${ }^{*}<0.0001$ (t-test); $n=4$. (D) Bar graph showing the percent of cells stained for both HLA and Ki67 in the wound center or periphery; ${ }^{*} p<0.0001$ (t-test); $n=3$. (E) Immunofluorescence of excised wounds showing immunoreactivity for HLA (green) as well as for SMA (red, left), elastin (red, middle), or SM-MHC (red, right); DAPI, blue; scale bar, $30 \mu \mathrm{m}$; yellow arrows show positive cells. (F) Bar graph showing the percent of HLA-positive cells also showing immunoreactivity with SMA, elastin, or SM-MHC; $\mathrm{n}=2-4$. (G) Immunofluorescence of excised wounds showing immunoreactivity for HLA (green) as well as for L-caldesmon (red, left) or vimentin (red, right); DAPI, blue; scale bar, $30 \mu \mathrm{m}$; yellow arrows denote positive cells. (H) Bar graph showing the percent of HLA-positive cells also showing immunoreactivity with $\mathrm{L}$-caldesmon or vimentin; $\mathrm{n}=2-4$.

DAPI: 4',6-diamidino-2-phenylindole; SM-MHC: Smooth muscle-myosin heavy chain; SMA: Smooth muscle actin.

with increased numbers of total macrophages, with decreased numbers of M1-type macrophages and increased numbers of M2-type macrophages.

\section{Delivery of hiPSC-SMC in collagen scaffolds promotes cellular retention in vivo}

Since delivery of MSC in a 3D collagen scaffold increased their survival in vivo [30], we determined whether the scaffold has a similar effect on hiPSC-SMC. At 7 days, hiPSC-SMC are present in the wound and are concentrated in the wound center $(\mathrm{p}<0.0001$; Figure $6 \mathrm{~A} \& \mathrm{C}$ ). A total of $73 \%$ of the hiPSC-SMC in the wound center were Ki67-positive compared with $2 \%$ in the wound periphery ( $p<0.0001$; Figure $6 \mathrm{~B} \& \mathrm{D})$, consistent with 
(A)

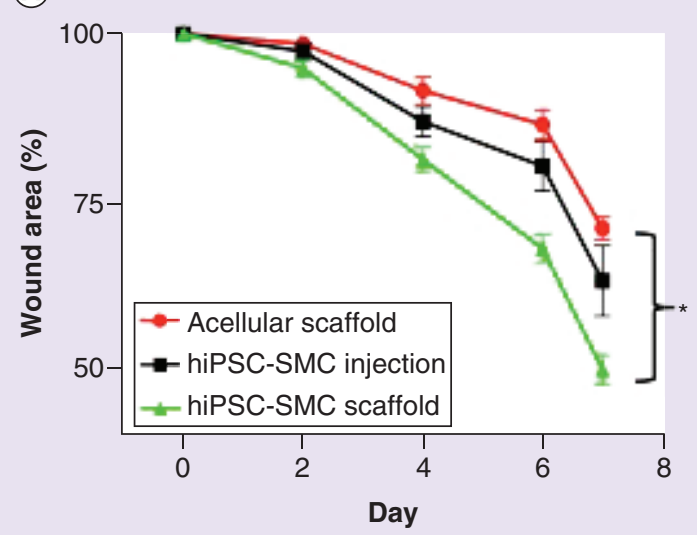

(B)
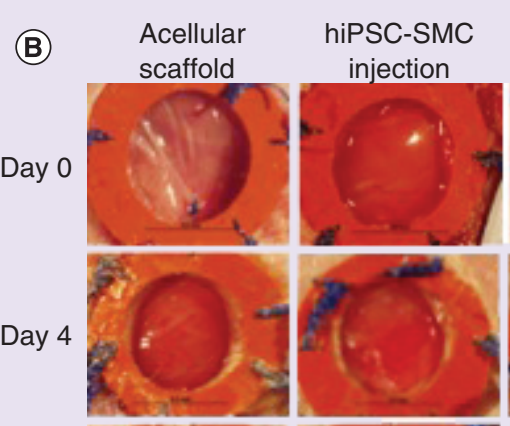

Day 7

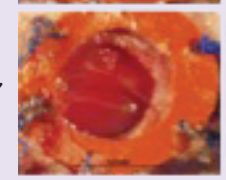

hiPSC-SMC scaffold
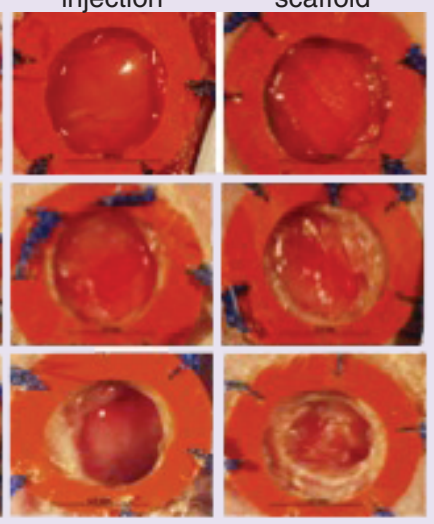

(C)
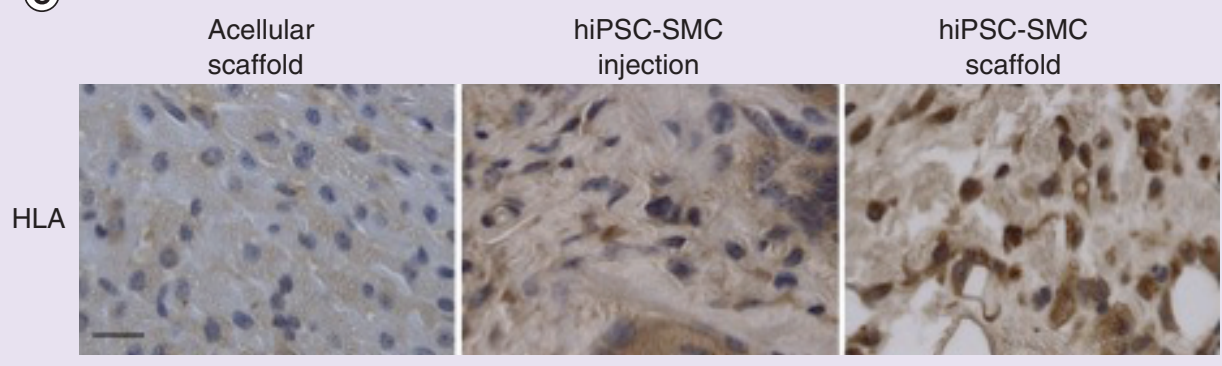

(D)

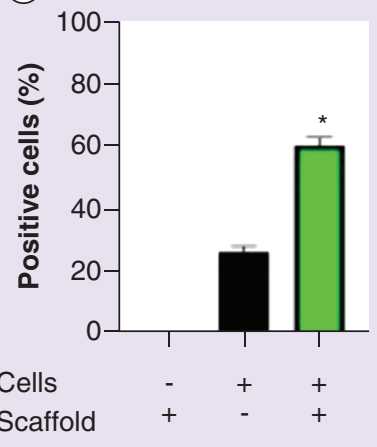

Figure 7. Delivery of cells in a collagen scaffold accelerates wound healing and increases cell retention. (A) Line graph representing wound area over time in acellular scaffolds, directly injected hiPSC-SMC and hiPSC-SMC delivered in the scaffold; * $p<0.0001$ (two-way ANOVA); $p=0.0168$ at day 2 hiPSC-SMC injection vs hiPSC-SMC scaffold; $p=0.0022$ at day 4 hiPSC-SMC injection vs hiPSC-SMC scaffold; $\mathrm{p}=0.0003$ at day 6 hiPSC-SMC injection vs hiPSC-SMC scaffold; $\mathrm{p}=0.0022$ at day 7 hiPSC-SMC injection vs hiPSC-SMC scaffold; (Tukey's post hoc); $\mathrm{n}=6$. (B) Photographs of representative wounds at days 0, 4 and 7 in acellular, hiPSC-SMC injection and hiPSC-SMC scaffold groups; scale bar, $5 \mathrm{~mm}$. (C) Photomicrographs of excised wounds stained for HLA, in the hiPSC-SMC injection (left) and hiPSC-SMC scaffold (right) groups; scale bar, $10 \mu \mathrm{m}$. (D) Bar graph showing the percent of HLA-positive cells in the wound in acellular hiPSC-SMC injection and hiPSC-SMC scaffold groups; * $p<0.0001$ (one-way ANOVA); $p<0.0001$ hiPSC-SMC injection vs hiPSC-SMC scaffold (Tukey's post hoc); $n=4$; -: no; +: yes.

ANOVA: Analysis of variance; hiPSC-SMC: Human-induced pluripotent stem cell-derived smooth muscle cell.

a proliferative SMC phenotype. Similarly, few hiPSC-SMC were SMA, elastin or SM-MHC-positive (Figure 6E $\& \mathrm{~F}$ ), whereas cells showed a high percentage of caldesmon light chain and vimentin immunoreactivity (Figure 6G $\& \mathrm{H})$.

To confirm that delivery of hiPSC-SMC in a collagen scaffold improves cellular survival and accelerates wound healing, we compared the wound healing rate in wounds treated with acellular scaffolds, directly injected hiPSCSMC without a scaffold and hiPSC-SMC delivered within the scaffold. Delivery of hiPSC-SMC in collagen scaffolds accelerated wound healing to a greater extent than acellular scaffolds as well as compared with hiPSCSMC that were directly injected ( $<$ 0.0001; Figure 7A \& B); 7 days post treatment, the iPSC-SMC scaffold group was also associated with a higher percentage of surviving HLA-positive hiPSC-SMC ( $<<0.0001$; Figure 7C \& D). These data suggest that delivery of hiPSC-SMC in a 3D scaffold improves their survival in vivo, with synthetic phenotype hiPSC-SMC detectable in the wound center. 


\section{Discussion}

This study shows that hiPSC differentiated into SMC in vitro have a predominantly contractile phenotype (Figure 1) and secrete more cytokines inside the hypoxic environment of rolled 3D collagen scaffolds compared with flat scaffolds (Figure 2); hiPSC-SMCs also secrete increased concentration of angiogenic and regenerative cytokines compared with ADSC and the rolled configuration leads to increased expression of HIF-1 $\alpha$ (Figure 2). hiPSC-SMC accelerate diabetic wound healing in a full thickness splinted nude mouse wound model compared with acellular scaffolds and ADSC-containing scaffolds (Figure 3) and are associated with increased cellular proliferation, VEGF$A$ and bFGF expression, as well as increased angiogenesis (Figure 4). After 7 days, there are also increased number of overall and M2-type macrophages, with reduced numbers of M1-type macrophages (Figure 5). At the same time point, wounds treated with hiPSC-SMC have retained cells that proliferate and display a predominantly synthetic phenotype (Figure 6). hiPSC-SMC survived in vivo for 7 days which was increased compared with directly injected cells (Figure 7). These data suggest that hiPSC-SMC are able to accelerate diabetic wound healing in a mouse model, suggesting their translational potential to human patients with DFU.

Our primary finding shows that hiPSC-SMC secrete increased concentrations of angiogenic cytokines and accelerate diabetic wound healing to a greater extent than acellular collagen scaffolds and ADSC-containing scaffolds (Figures $2 \& 3$ ). It has previously been shown that SMC promote endothelial cell neovascularization and wound healing $[37,38]$. In addition, during wound healing, SMC serve as a source of myofibroblasts, cells essential to granulation tissue formation and that are downregulated in chronic and diabetic wounds [39,40]. One study showed that vascular SMC obtained from the skin of diabetic donors were fewer in number and associated with impaired wound closure in vitro, compared with SMC derived from healthy donors, suggesting that SMC serve several roles in healing, particularly in diabetic wounds [41]. Although hiPSC-derived EC, MSC and fibroblasts accelerate wound healing in murine models, the wound healing potential of hiPSC-SMC alone has not been reported, despite improved hiPSC-SMC differentiation protocols compared with hiPSC-EC [20,22-24,27,28]. Here, we present the first report of hiPSC-SMC accelerating diabetic wound healing in an in vivo model.

Our data also show that treatment of diabetic wounds with hiPSC-SMC is associated with increased numbers of overall and M2-type macrophages, with a reduced numbers of M1-type macrophages (Figure 5). Global macrophage depletion leads to impaired wound healing and is associated with increased pro-inflammatory cytokines, reduced myofibroblast differentiation and wound contracture, as well as temporal dysregulation of VEGF signaling and resultant decreased angiogenesis [42,43]. In addition, chronic wounds are associated with a failure of M1-M2-type macrophage switching, resulting in prolonged inflammation, reduction in growth factors and angiogenesis as well as excessive extracellular matrix proteolysis and chronic nonhealing [44-48]. The success of MSC in treating chronic wounds may be due to their immunomodulatory effects, which leads to decreased inflammation in the wound bed [49]. Our findings of decreased M1-type and increased M2-type macrophages in hiPSC-SMC-treated wounds suggest that hiPSC-SMC treatment increases the total number of macrophages or shifts the M1-M2 phenotypic switch at an earlier time point, compared with acellular and ADSC-containing scaffolds.

Although our study is the first to show that hiPSC-SMC accelerate diabetic wound healing in a murine model, there are several limitations. While commonly used, the streptozotocin model of diabetes is a closer model of Type 1 diabetes, whereas Type 2 diabetes is more frequently seen in patients suffering from chronic wounds and diabetic foot ulcers. In addition, a major obstacle to clinical translation of iPSC therapy into human patients is the teratogenic potential of undifferentiated cells [50]. Although there has been no observed teratoma formation after topical iPSC application, iPSC injected subcutaneously retain their teratogenic potential [51]. Once transplanted onto the wound bed, iPSC-SMC transition from a contractile to synthetic and proliferative phenotype, raising concern for their de-differentiation and hyperplastic potential. In addition, this study only examined male animals since male patients with DFU are more likely to require lower extremity amputations compared with women; further studies in female animals are necessary to validate these findings [52]. At last, although our study shows that hiPSC-SMC are associated with improved wound healing compared with ADSC, the mechanism for the improved healing remains to be determined, especially since both cell types are detectable in the healing wound through day 7 (Figures 6 \& 7) [30]. Despite these limitations, our study identifies hiPSC-SMC as a potential cellular source to accelerate diabetic wound healing.

\section{Conclusion}

Our work is the first to show that hiPSC-SMC accelerate diabetic wound healing when delivered alone in our previously validated rolled collagen scaffold model. Similar to our previously studied murine ADSC controls, 
hiPSC-SMC are activated by the hypoxic environment of a rolled scaffold, which results in release of increased quantities of pro-angiogenic cytokines [30]. In vivo, hiPSC-SMC accelerate diabetic wound healing in a splinted nude mouse model, compared with acellular controls. hiPSC-SMC treatment is also associated with increased cellular proliferation and improved dermal architecture and growth factor concentrarions. At last, hiPSC-SMC-treated wounds have increased total and M2-type macrophages as well as greater wound neovascularization.

\section{Translational perspective}

Induced pluripotent stem cells are a new and exciting cellular type in regenerative medicine. iPSC are pluripotent and therefore potentially capable of repopulating any cellular lineage and they are not associated with the ethical dilemmas surrounding embryonic stem cell therapy. Multiple iPSC-derived cell lines, including ECs and MSCs, show wound healing potential. Despite efficient protocols to differentiate iPSC-SMC, thus far, no study has examined the use of iPSC-SMC in wound healing [18]. Our study is the first to show that iPSC-SMC delivered alone accelerate diabetic wound healing.

Despite promising animal studies, no iPSC-derived cells have been used in clinical trials for diabetic wound healing, due to their safety profile limitations. Among other concerns, integrative methods of inducing pluripotency have the potential to damage host DNA and undifferentiated cells have tumorigenic potential. Although the safety profile of iPSC-derived cells has been examined following injections, safety of topical iPSC application has not been studied. Consideration for clinical translation requires further understanding of the long-term potential for hyperplastic growth if even a small number of hiPSC-SMC become incorporated into the healed wound.

In order to transition iPSC technology from the bench to bedside, nonintegrative means of inducing pluripotency must be examined in close detail, methods of increasing differentiation efficiency must be improved, ways of eliminating undifferentiated cells must be perfected and safety after topical iPSC-derived cellular application must be examined.

\section{Summary points}

- Induced pluripotent stem cell-derived smooth muscle cells (iPSC-SMC) display a contractile phenotype in vitro.

- iPSC-SMC secrete increased amounts of pro-angiogenic and growth cytokines when compared with murine adipose-derived stem cells (ADSC) in vitro.

- iPSC-SMC delivered in collagen scaffolds accelerate diabetic wound healing in a splinted, nude mouse model and compared with directly injected cells.

- iPSC-SMC treatment is associated with improved skin morphology, compared with control and murine ADSC-containing scaffolds.

- Wounds treated with iPSC-SMC are associated with increased proliferation, pro-angiogenic cytokine expression and angiogenesis.

- iPSC-SMC undergo a phenotype switch to synthetic forms and proliferate in the wound bed following treatment.

- iPSC-SMC treatment is associated with increased overall number of macrophages, with less M1 type and more M2 type macrophages, compared with acellular and murine ADSC wounds.

\section{Author contributions}

Designing of research studies was conducted by J Gorecka, A Fereydooni, BC Dash, J Luo, SR Lee, R Taniguchi, X Gao, HC Hsia, Y Qyang and A Dardik. J Gorecka, A Fereydooni, BC Dash, J Luo, SR Lee, R Taniguchi and X Gao conducted the experiments. J Gorecka, A Fereydooni, BC Dash, J Luo, SR Lee, R Taniguchi and X Gao acquired the data. J Gorecka, A Fereydooni and BC Dash analyzed the data. J Gorecka, A Fereydooni, BC Dash, J Luo, SR Lee, R Taniguchi, X Gao, HC Hsia, Y Qyang and A Dardik prepared the manuscript.

\section{Financial \& competing interests disclosure}

This work was supported by the Association of VA Surgeons Resident Research Award (J Gorecka), Howard Hughes Medical Institute Fellowship and Society of Vascular Surgery Research Fellowship (A Fereydooni), Plastic Surgery Foundation Grant 18-003032 (BC Dash), the Plastic Surgery Foundation (HC Hsia), the National Institutes of Health R01HL116705 and Connecticut's Regenerative Medicine Research Fund (CRMRF) (Y Qyang), the NIH R01-HL128406 (A Dardik), as well as with the resources and the use of facilities at the VA Connecticut Healthcare System, West Haven, CT. The authors have no other relevant affiliations or financial involvement with any organization or entity with a financial interest in or financial conflict with the subject matter or materials discussed in the manuscript apart from those disclosed.

No writing assistance was utilized in the production of this manuscript. 


\section{Ethical conduct of research}

The authors state that they have obtained appropriate institutional review board approval or have followed the principles outlined in the Declaration of Helsinki for all human or animal experimental investigations.

\section{Open access}

This work is licensed under the Attribution-NonCommercial-NoDerivatives 4.0 Unported License. To view a copy of this license, visit http://creativecommons.org/licenses/by-nc-nd/4.0/

\section{References}

1. American Diabetes Association. Economic costs of diabetes in the U.S. in 2012. Diabetes Care 36(4), 1033-1046 (2013).

2. Prevention CFDCA. National diabetes statistics report, 2017 (2017). www.cdc.gov/diabetes/data/statistics/statistics-report.html

3. Armstrong DG, Boulton AJM, Bus SA. Diabetic foot ulcers and their recurrence. N. Engl. J. Med. 376(24), 2367-2375 (2017).

4. Frykberg RG, Zgonis T, Armstrong DG et al. Diabetic foot disorders: a clinical practice guideline (2006 revision). J. Foot Ankle Surg. 45(Suppl. 5), S1-S66 (2006).

5. Cavanagh PR, Lipsky BA, Bradbury AW, Botek G. Treatment for diabetic foot ulcers. Lancet 366(9498), 1725-1735 (2005).

6. Falanga V. Wound healing and its impairment in the diabetic foot. Lancet 366(9498), 1736-1743 (2005).

7. Gallagher KA, Liu Z-J, Xiao M et al. Diabetic impairments in NO-mediated endothelial progenitor cell mobilization and homing are reversed by hyperoxia and SDF-1 alpha. J. Clin. Invest. 117(5), 1249-1259 (2007)

8. Galkowska H, Wojewodzka U, Olszewski WL. Chemokines, cytokines and growth factors in keratinocytes and dermal endothelial cells in the margin of chronic diabetic foot ulcers. Wound Repair Regen. 14(5), 558-565 (2006).

9. Maruyama K, Asai J, Ii M, Thorne T, Losordo DW, D’amore PA. Decreased macrophage number and activation lead to reduced lymphatic vessel formation and contribute to impaired diabetic wound healing. Am. J. Pathol. 170(4), 1178-1191 (2007).

10. Isakson M, De Blacam C, Whelan D, Mcardle A, Clover AJP. Mesenchymal stem cells and cutaneous wound healing: current evidence and future potential. Stem Cells Int. 2015, 831095-831095 (2015).

11. Cao Y, Gang X, Sun C, Wang G. Mesenchymal stem cells improve healing of diabetic foot ulcer. J. Diabetes Res. 2017, 9328347-9328347 (2017).

12. Mizukami H, Yagihashi S. Exploring a new therapy for diabetic polyneuropathy - the application of stem cell transplantation. Front. Endocrinol. (Lausanne) 5, 45-45 (2014).

13. Lu D, Chen B, Liang Z et al. Comparison of bone marrow mesenchymal stem cells with bone marrow-derived mononuclear cells for treatment of diabetic critical limb ischemia and foot ulcer: a double-blind, randomized, controlled trial. Diabetes Res. Clin. Pract. 92(1), 26-36 (2011).

14. Xu S-M, Liang T. Clinical observation of the application of autologous peripheral blood stem cell transplantation for the treatment of diabetic foot gangrene. Exp. Ther. Med. 11(1), 283-288 (2016).

15. Kirana S, Stratmann B, Prante C et al. Autologous stem cell therapy in the treatment of limb ischaemia induced chronic tissue ulcers of diabetic foot patients. Int. J. Clin. Pract. 66(4), 384-393 (2012).

16. Gadelkarim M, Abushouk AI, Ghanem E, Hamaad AM, Saad AM, Abdel-Daim MM. Adipose-derived stem cells: effectiveness and advances in delivery in diabetic wound healing. Biomed. Pharmacother. 107, 625-633 (2018).

17. Balaji S, Keswani SG, Crombleholme TM. The role of mesenchymal stem cells in the regenerative wound healing phenotype. Adv Wound Care 1(4), 159-165 (2012).

18. Gorecka J, Kostiuk V, Fereydooni A et al. The potential and limitations of induced pluripotent stem cells to achieve wound healing. Stem Cell. Res. Ther. 10(1), 87-87 (2019).

19. Kirby GT, Mills SJ, Cowin AJ, Smith LE. Stem cells for cutaneous wound healing. Biomed Res. Int. 2015, 285869 (2015).

20. Clayton ZE, Tan RP, Miravet MM et al. Induced pluripotent stem cell-derived endothelial cells promote angiogenesis and accelerate wound closure in a murine excisional wound healing model. Biosci. Rep. 38(4), BSR20180563 (2018).

21. Clayton ZE, Yuen GS, Sadeghipour S et al. A comparison of the pro-angiogenic potential of human induced pluripotent stem cell derived endothelial cells and induced endothelial cells in a murine model of peripheral arterial disease. Int. J. Cardiol. 234, 81-89 (2017).

22. Tan RP, Chan AHP, Lennartsson K et al. Integration of induced pluripotent stem cell-derived endothelial cells with polycaprolactone/gelatin-based electrospun scaffolds for enhanced therapeutic angiogenesis. Stem Cell. Res. Ther. 9(1), 70-70 (2018).

23. Kashpur O, Smith A, Gerami-Naini B et al. Differentiation of diabetic foot ulcer-derived induced pluripotent stem cells reveals distinct cellular and tissue phenotypes. FASEB J. 33(1), 1262-1277 (2018).

24. Nakayama C, Fujita Y, Matsumura W et al. The development of induced pluripotent stem cell-derived mesenchymal stem/stromal cells from normal human and RDEB epidermal keratinocytes. J. Dermatol. Sci. 91(3), 301-310 (2018). 
25. Kobayashi H, Ebisawa K, Kambe $\mathrm{M}$ et al. Editors' choice effects of exosomes derived from the induced pluripotent stem cells on skin wound healing. Nagoya J. Med. Sci. 80(2), 141-153 (2018).

26. Zhang J, Guan J, Niu X et al. Exosomes released from human induced pluripotent stem cells-derived MSCs facilitate cutaneous wound healing by promoting collagen synthesis and angiogenesis. J. Transl. Med. 13, 49 (2015).

27. Kim KL, Song S-H, Choi K-S, Suh W. Cooperation of endothelial and smooth muscle cells derived from human induced pluripotent stem cells enhances neovascularization in dermal wounds. Tissue Eng. Part A 19(21-22), 2478-2485 (2013).

28. Shen Y-I, Cho H, Papa AE et al. Engineered human vascularized constructs accelerate diabetic wound healing. Biomaterials 102, 107-119 (2016).

29. Klein D. iPSCs-based generation of vascular cells: reprogramming approaches and applications. Cell. Mol. Life Sci. 75(8), 1411-1433 (2018).

30. Assi R, Foster TR, He H et al. Delivery of mesenchymal stem cells in biomimetic engineered scaffolds promotes healing of diabetic ulcers. Regen. Med. 11(3), 245-260 (2016).

31. Guo J, Hu H, Gorecka J et al. Adipose-derived mesenchymal stem cells accelerate diabetic wound healing in a similar fashion as bone marrow-derived cells. Am. J. Physiol. Cell Physiol. 315(6), C885-C896 (2018).

32. Zhang P, Lu J, Jing Y, Tang S, Zhu D, Bi Y. Global epidemiology of diabetic foot ulceration: a systematic review and meta-analysis. Ann. Med. 49(2), 106-116 (2017).

33. Galiano RD, Michaels VJ, Dobryansky M, Levine JP, Gurtner GC. Quantitative and reproducible murine model of excisional wound healing. Wound Repair Regen. 12(4), 485-492 (2004).

34. Gui L, Dash BC, Luo J et al. Implantable tissue-engineered blood vessels from human induced pluripotent stem cells. Biomaterials 102, 120-129 (2016).

35. Dash BC, Levi K, Schwan J et al. Tissue-engineered vascular rings from human iPSC-derived smooth muscle cells. Stem Cell Reports 7(1), 19-28 (2016).

36. Luo J, Qin L, Kural MH et al. Vascular smooth muscle cells derived from inbred swine induced pluripotent stem cells for vascular tissue engineering. Biomaterials 147, 116-132 (2017).

37. Kuzuya M, Satake S, Esaki T et al. Induction of angiogenesis by smooth muscle cell-derived factor: possible role in neovascularization in atherosclerotic plaque. J. Cell. Physiol. 164(3), 658-667 (1995).

38. Foubert P, Squiban C, Holler V et al. Strategies to enhance the efficiency of endothelial progenitor cell therapy by ephrin B2 pretreatment and coadministration with smooth muscle progenitor cells on vascular function during the wound-healing process in irradiated or nonirradiated condition. Cell Transplant 24(7), 1343-1361 (2015).

39. Hinz B. Myofibroblasts. Exp. Eye Res. 142, 56-70 (2016).

40. Hinz B. The role of myofibroblasts in wound healing. Curr. Res. Transl. Med. 64(4), 171-177 (2016).

41. Steinbach Sarah K, Yau Terrence M, Ouzounian M et al. Skin-derived precursors from human subjects with Type 2 diabetes yield dysfunctional vascular smooth muscle cells. Clin. Sci. 131(15), 1801 (2017).

42. Goren I, Allmann N, Yogev N et al. A transgenic mouse model of inducible macrophage depletion: effects of diphtheria toxin-driven lysozyme M-specific cell lineage ablation on wound inflammatory, angiogenic and contractive processes. Am. J. Pathol. 175(1), 132-147 (2009).

43. Hesketh M, Sahin KB, West ZE, Murray RZ. Macrophage phenotypes regulate scar formation and chronic wound healing. Int. J. Mol. Sci. 18(7), 1545 (2017).

44. Loots MaM, Lamme EN, Zeegelaar J, Mekkes JR, Bos JD, Middelkoop E. Differences in cellular infiltrate and extracellular matrix of chronic diabetic and venous ulcers versus acute wounds. J. Invest. Dermatol. 111(5), 850-857 (1998).

45. Sindrilaru A, Peters T, Wieschalka $S$ et al. An unrestrained proinflammatory M1 macrophage population induced by iron impairs wound healing in humans and mice. J. Clin. Invest. 121(3), 985-997 (2011).

46. Miao M, Niu Y, Xie T, Yuan B, Qing C, Lu S. Diabetes-impaired wound healing and altered macrophage activation: a possible pathophysiologic correlation. Wound Repair Regen. 20(2), 203-213 (2012).

47. Okizaki S-I, Ito Y, Hosono K et al. Suppressed recruitment of alternatively activated macrophages reduces TGF- $\beta 1$ and impairs wound healing in streptozotocin-induced diabetic mice. Biomed. Pharmacother. 70, 317-325 (2015).

48. Subramaniam K, Pech CM, Stacey MC, Wallace HJ. Induction of MMP-1, MMP-3 and TIMP-1 in normal dermal fibroblasts by chronic venous leg ulcer wound fluid. Int. Wound J. 5(1), 79-86 (2008).

49. Regulski M. Mesenchymal stem cells: "guardians of inflammation". Wounds 29(1), 20-27 (2017).

50. Gutierrez-Aranda I, Ramos-Mejia V, Bueno C et al. Human induced pluripotent stem cells develop teratoma more efficiently and faster than human embryonic stem cells regardless the site of injection. Stem Cells 28(9), 1568-1570 (2010).

51. Roy NS, Cleren C, Singh SK, Yang L, Beal MF, Goldman SA. Functional engraftment of human ES cell-derived dopaminergic neurons enriched by coculture with telomerase-immortalized midbrain astrocytes. Nat. Med. 12, 1259 (2006).

52. Sayiner ZA, Can FI, Akarsu E. Patients' clinical charecteristics and predictors for diabetic foot amputation. Prim. Care Diabetes 13(3), 247-251 (2019). 